EESTI NSV TEADUSTE AKADEEMIA TOIMETISED. 28. KOIDE

FOOSIKA * MATEMAATIKA. 1979, NR. 1

ИЗВЕСТИЯ АКАДЕМИИ НАУК ЭСТОНСКОИ ССР. ТОМ 28 ФИЗИКА * МАТЕМАТИКА. 1979, № 1

\title{
О НЕКОТОРЫХ ЗАДАЧАХ СВОБОДНОЙ ТУРБУЛЕНТНОСТИ
}

\author{
(Представлена И. Эпиком)
}

Задачи о развитии свободной турбулентной струи или следа на основном участке течения принадлежат к числу классических в теории пограничного слоя. Их решения на основе ранних феноменологических моделей турбулентности Прандтля, Тейлора и др. $\left[{ }^{1-3}\right]$ дают возможность получить только осредненные характеристики течения. Хорошо известны и другие недостатки этих решений, в частности определенные отклонения расчетных кривых и опытных данных вблизи оси симметрии или у внешнего края течения. Попытка расчета пульсационных характеристик течения применительно к вышеупомянутым задачам с привлечением уравнения баланса турбулентной энергии была предпринята в $\left[{ }^{4}\right]$.

Ниже приводятся расчеты плоской затопленной струи и плоского следа в несжимаемой жидкости на основе модели, развитой в $\left[{ }^{5}\right]$.

\section{1. Плоская струя}

Исходные уравнения неразрывности, переноса импульса и баланса турбулентной энергии первичной составляющей пульсации скорости применительно к рассматриваемой задаче имеют следующий вид:

$$
\begin{aligned}
\partial U / \partial x+\partial V / \partial y & =0 \\
U(\partial U / \partial x)+V(\partial U / \partial y) & =\partial[\varepsilon(\partial U / \partial y)] / \partial y \\
U\left(\partial q_{0}^{2} / \partial x\right)+V\left(\partial q_{0}^{2} / \partial y\right) & =\frac{1}{A} \partial\left[\varepsilon\left(\partial q_{0}^{2} / \partial y\right)\right] / \partial y-B\left(q_{0}^{3} / l\right)
\end{aligned}
$$

где $U, V$ - компоненты осредненной скорости по осям $x, y, q_{0}$ - модуль вектора первичной составляющей пульсации скорости, $l$ - линейный масштаб турбулентности, $A, B$ - константы, $\varepsilon-$ коэффициент турбулентной вязкости, равный

$$
\varepsilon=N q_{0} l
$$

при $N=1 / 3$, т. е. предполагается равная вероятность турбулентных пульсаций в любом направлении.

Граничные условия и интегральное условие сохранения импульса имеют вид

$$
\begin{array}{cc}
\partial U / \partial y=V=\partial q_{0}^{2} / \partial y=0 & \text { при } \quad y=0, \\
U=q_{0}^{2} \rightarrow 0 & \text { при } y \rightarrow \infty,
\end{array}
$$




$$
\int_{0}^{\infty} U^{2} d y=I
$$

где $I$ - начальный импульс струн.

Исходя из принятого в $\left[{ }^{5}\right]$ допущения $l / a=$ const $(a-$ характерная ширина пограничного слоя) и учитывая, что в автомодельном режиме $a \sim x$, имеем

$$
l=k x,
$$

где $k-$ константа.

Введем новые переменные:

$$
\begin{gathered}
U=(I / x x)^{1 / 2} F^{\prime}(\varphi), \quad V=(x I / x)^{1 / 2}\left[\varphi F^{\prime}(\varphi)-F(\varphi) / 2\right], \\
q_{0}=(3 I / x x)^{1 / 2} \chi f(\varphi),
\end{gathered}
$$

где $F, f$ - безразмерные функции переменной $\varphi=y / x x, x^{2}=2 k \chi / 3^{1 / 2}$, $\chi=\overline{u_{0 m}^{2}} / U_{m}=\overline{v_{0 m}^{2}} / U_{m}=\overline{w_{0 m}^{2}} / U_{m}$ - интенсивность турбулентности на оси струи, $U_{m}$ - осредненная скорость на оси струи.

Преобразование соотношений $(1.2),(1.3)$, (1.5) с помощью (1.8) и с учетом (1.4), (1.7) приводит к следующей системе уравнений:

$$
\begin{aligned}
& F F^{\prime}+f F^{\prime \prime}=0, \\
& f F^{\prime}+f^{\prime} F+\frac{1}{A}\left(f f^{\prime \prime}+2 f^{\prime 2}\right)-B_{1} f^{2}=0, \\
& \left(B_{1}=3^{1 / 2} \chi B / k\right)
\end{aligned}
$$

с граничными условиями

$$
\begin{array}{ll}
F=f^{\prime}=0, F^{\prime}=f=1 & \text { при } \quad \varphi=0, \\
F^{\prime}=f \rightarrow 0 & \text { при } \quad \varphi \rightarrow \infty .
\end{array}
$$

Интегральное условие однозначности

$$
\int_{0}^{\infty} F^{\prime 2} d \varphi=1
$$

может служить для контроля полученного решения.

Система (1.9), (1.10) решалась численно методом Рунге-Кутта при значениях константы $A=0,5 ; 0,75$ и 1,0 . Численное значение $B_{1}=0,43$ было подобрано таким образом, чтобы для $f$ удовлетворялись граничные условия (1.11) на бесконечности (практически же на некотором конечном расстоянии $\varphi=2,5-3)$.

Сравнение результатов расчета с опытными данными $\left[{ }^{6-8}\right]$ по средней продольной скорости показало их хорошее в целом соответствие (см. pис. 1 , где $y_{0,5}$ - поперечное расстояние, $\left.U=0,5 U_{m}\right)$. Изменение $A$ в пределах $0,5-1,0$ оказывает заметное влияние на расчеты лишь вблизи внешней границы течения, что, однако, при наблюдаемом разбросе самих опытных точек не имеет существенного значения.

Численное значение $\chi$ (определенное по среднеарифметическому от фактически измеренных значений $\overline{u^{2}}, \overline{v^{2}}$ и $\overline{w^{2}}$ на оси струи) оказалось равным 0,212 в $\left[{ }^{7}\right]$ и 0,224 в $\left[{ }^{8}\right]$ при $y_{0,5} / x=0,109$. Тогда при $A=0,75$ получим следующие значения констант: $B=0,043, \quad x=0,0945, k=$ $=0,0365$ для $\left[{ }^{7}\right]$ или $B=0,038, x=0,0945, k=0,0345$ для [ $\left.{ }^{8}\right]$ (чему 
соответствует в среднем $l / a \approx$ $\approx 0,16)$. При других значениях $A$ величины констант отличаются от вышеприведенных также незначительно.

С учетом (1.2), (1.4), (1.8) получим следующую формулу для турбулентных касательных напряжений

$$
-\overline{u v} / U_{m}^{2}=x f F^{\prime \prime} / 2=x F F^{\prime} / 2
$$

Результаты расчета профиля касательных напряжений достаточно хорошо согласуются с соответствующими опытными данными $\left[{ }^{7}\right]$, но оказываются несколько завышенными относительно данных $\left[{ }^{8}\right]$ в зоне максимальных значений $\overline{u v}$ и во внешней части течения (рис. 2), что можно объяснить неучетом фактора перемежаемости.

В $\left[{ }^{5}\right]$ были получены формулы для среднеквадратичных значений пульсации скорости, которые в случае свободного пограничного слоя принимают вид

$$
\begin{aligned}
& \overline{u^{2}}=\left[q_{0}^{2}+l^{2}(\partial U / \partial y)^{2}\right] / 3 \\
& \overline{v^{2}}=\overline{w^{2}}=q_{0}^{2} / 3
\end{aligned}
$$

Тогда, учитывая (1.8), имеем

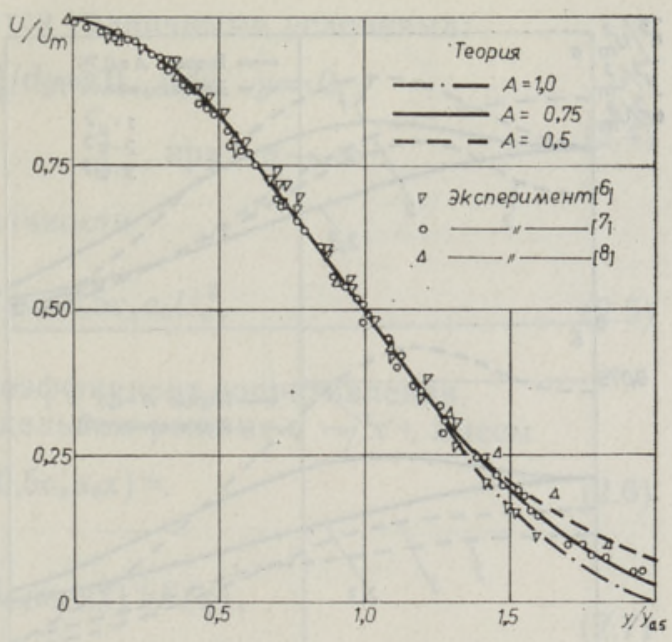

HHC. 1.

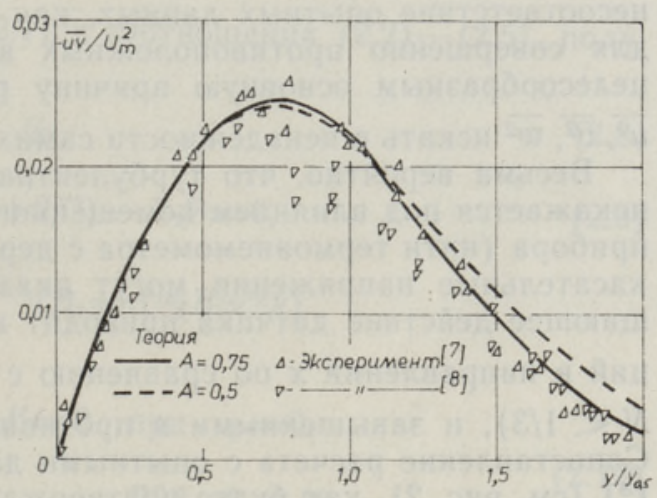

Рис. 2.

$$
\overline{u^{2} / U_{m}^{2}}=\chi^{2} f^{2}+\left(k F^{\prime \prime} / \chi\right)^{2} / 3, \quad \overline{v^{2}} U_{m}^{2}=\overline{w^{2}} / U_{m}^{2}=\chi^{2} f^{2} .
$$

При сопоставлении расчета с фактически измеренными значениями $\overline{u^{2}}, \overline{v^{2}}, \overline{w^{2}}$ (рис. 3) обращает на себя внимание следующее. Из принятого допущения о равной вероятности турбулентного переноса в любом направлении следует равенство $\overline{u_{m}^{2}}=\overline{v_{m}^{2}}=\overline{w_{m}^{2}}$. Опытные же данные как будто не подтверждают этого. Однако бросается в глаза явная противоречивость этих данных. Так, в опытах $\left[{ }^{7}\right]$ с плоской струей $\overline{u_{m}^{2}} / \overline{v_{m}^{2}} \approx 0,76$ и $\overline{u_{m}^{2} / w_{m}^{2}} \approx 1,35$, а в опытах [ $\left.{ }^{9}\right]$ с плоским следом $\overline{u_{m}^{2}} / \overline{v_{m}^{2}} \approx 0,72$ и $\overline{u_{m}^{2} / w_{m}^{2}} \approx 1,07$. С другой стороны, в опытах [ $\left.{ }^{8}\right]$ с плоской струей $\overline{u_{m}^{2} / v_{m}^{2}} \approx 2$ и $\overline{u_{m}^{2} / w_{m}^{2}} \approx 1,7$, а в опытах [10] с осесимметричной струей $\overline{u_{m}^{2}} \sqrt{v_{m}^{2}} \approx \overrightarrow{u_{m}^{2} / w_{m}^{2}} \approx 1,1$. При этом, однако, полная кинетическая энергия $\left(\overline{u_{m}^{2}}+\overline{v_{m}^{2}}+\overline{w_{m}^{2}}\right) / 2$ (а следовательно, и среднеариф- 


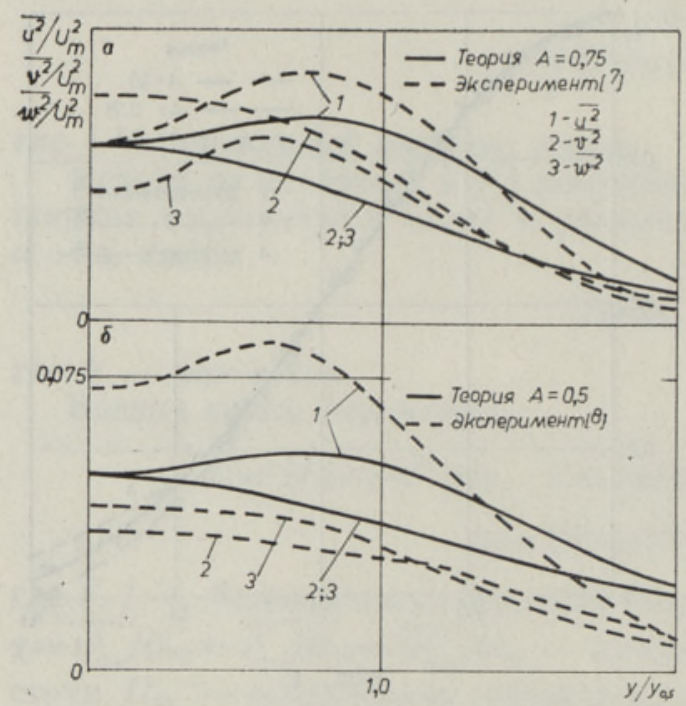

Рис 3.

метическое значение $\overline{u_{m}^{2}}, \overline{v_{m}^{2}}$, $\overline{\left.w_{m}^{2}\right)}$ в $[7,8]$ примерно одинакова. Характер расчетных кривых для $\overline{u^{2}}$ и $\overline{v^{2}}$ находится в качественном соответствии со всеми перечисленными опытными данными, хотя количественные расхождения, и весьма значительные, имеются и здесь. В отношении $\overline{w^{2}}$ существует качественное согласие с опытными данными $\left[{ }^{8,10}\right]$. В измерениях же $\left[{ }^{7,9}\right]$ наблюдался некоторый рост $\overline{w^{2}}$ в направлении от оси течения.

Трудно привести какие-либо доводы против допущения о равной вероятности турбулентного переноса в любом направлении, тем более, что несоответствие опытных данных, как уже указывалось, дает основание для совершенно противоположных выводов. Представляется поэтому целесообразным основную прнчину расхождения с экспериментом по $\overline{u^{2}}, \overline{v^{2}}, \overline{w^{2}}$ искать в ненадежности самих опытных данных.

Весьма вероятно, что турбулентная структура течения существенно искажается под влиянием помещенного в поток датчика измерительного прибора (нити термоанемометра с державкой). Согласно формуле (1.4), касательные напряжения могут оказаться заниженными, если возмущающее действие датчика приводит к увеличению вероятности пульсаций в направлении $x$ по сравнению с направлением $y$ (т. е. $\overline{u_{0}^{2}}>\overline{v_{0}^{2}}$ и $N<1 / 3)$, и завышенными в противном случае $\left(\overline{u_{0}^{2}}<\overline{v_{0}^{2}}\right.$ и $\left.N>1 / 3\right)$. Сопоставление расчета с опытными данными, в особенности с данными [ $\left.{ }^{8}\right]$ (см. рис. 2), как будто подтверждает такой вывод. Для окончательного решения вопроса, однако, необходимы измерения турбулентных характеристик неконтактными методами.

\section{2. Плоский след}

Для данного течения исходные уравнения имеют вид (с учетом (1.4)):

$$
\begin{gathered}
\partial U_{1} / \partial x+\partial V / \partial y=0 \\
U_{0}\left(\partial U_{1} / \partial x\right)=\frac{1}{3} \partial\left[q_{0} l\left(\partial U_{1} / \partial y\right)\right] / \partial y, \\
U_{0}\left(\partial q_{0}^{2} / \partial x\right)=\frac{1}{3 A} \partial\left[q_{0} l\left(\partial q_{0}^{2} / \partial y\right)\right] / \partial y-B\left(q_{0}^{3} / l\right) .
\end{gathered}
$$

Здесь $U_{1}=U-U_{0}, U_{0}-$ скорость невозмущенного потока, обтекающего образующий след цилиндр. Остальные обозначения сохраняют тот же смысл, что и в задаче о плоской струе. 
Система (2.1)-(2.3) дополняется граничными условиями:

$$
\begin{array}{lll}
\partial U_{1} / \partial y=V=\partial q_{0}^{2} / \partial y=0 & \text { при } & y=0, \\
U_{1}=q_{0}^{2} \rightarrow 0 & \text { при } \quad y \rightarrow \infty
\end{array}
$$

и интегральным условием однозначности

$$
U_{0} \int_{0}^{\infty} U_{1} d y=0,5 c_{x} a_{0} U_{0}^{2},
$$

где $a_{0}$ - радиус цилиндра, $c_{x}-$ коэффициент сопротивления.

Так как $l / a=$ const и в автомодельном режиме $a \sim x^{1 / 2}$, имеем

$$
l=k\left(0,5 c_{x} a_{0} x\right)^{1 / 2} .
$$

Положим

$$
\begin{aligned}
& U_{1}=U_{0}\left(0,5 c_{x} a_{0} / \chi x\right)^{1 / 2} F(\varphi), \\
& q_{0}=\chi U_{0}\left(1,5 c_{x} a_{0} / \chi x\right)^{1 / 2} f(\varphi),
\end{aligned}
$$

где

$$
\varphi=y /\left(0,5 x c_{x} a_{0} x\right)^{1 / 2}, \quad x^{3 / 2}=2 \chi k / 3^{1 / 2} .
$$

Подставляя переменные $(2.6)$ и (2.7) в соотношения $(2.2)-(2.5)$, получим следующие уравнения

$$
\begin{aligned}
& \varphi F+f F^{\prime}=0, \\
& \varphi f^{\prime}+f+\frac{1}{A}\left(f f^{\prime \prime}+2 f^{\prime 2}\right)-B_{1} f^{2}=0, \\
& \left(B_{1}=3^{1 / 2} \chi B / \chi^{1 / 2} k\right)
\end{aligned}
$$

с граничными условиями

$$
\begin{array}{lll}
F=f=1, \quad F^{\prime}=f^{\prime}=0 & \text { при } & \varphi=0, \\
F=f \rightarrow 0 & \text { при } & \varphi \rightarrow \infty
\end{array}
$$

и интегральным условием однозначности

$$
\int_{0}^{\infty} F d \varphi=1
$$

Полученные в результате численного решения $(2.8),(2.9)$ профили осредненной скорости и в этом случае слабо зависят от изменения $A$ в пределах 0,5-1 и хорошо согласуются с профилями, измеренными в $\left[{ }^{3,9}\right]$ (рис. 4). При этом $B_{1}=$ $=0,60$. В [ $\left.{ }^{9}\right]$ найдено $\chi=0,286$. Тогда при $A=0,75$ верно $\varkappa=$ $=0,196, k=0,264$ (чему соответствует $l / a \approx 0,23)$ и $B=$ $=0,141$.

Рис. 4.

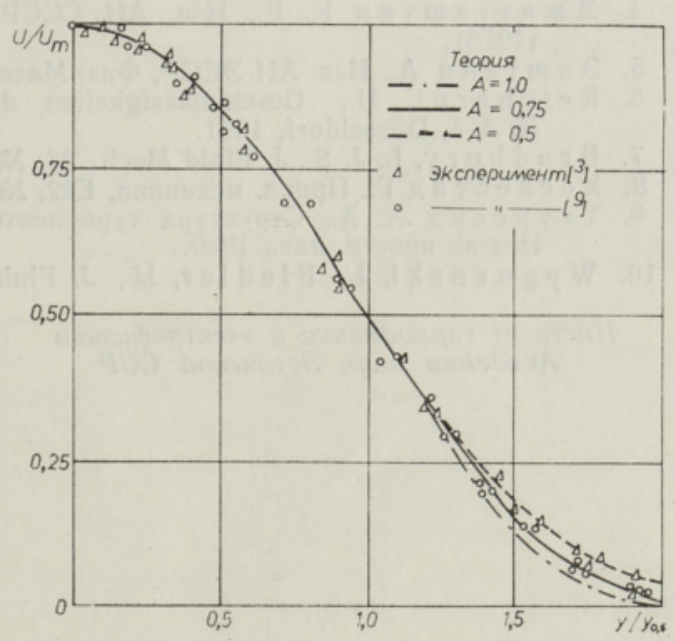






Рис. 5.

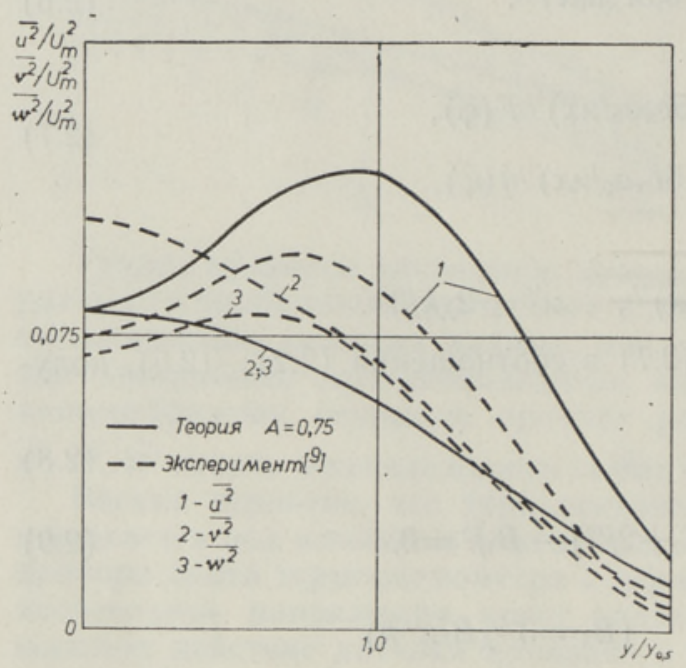

Рис. 6.
Формула для касательных напряжений имеет вид

$-\overline{u v} / U_{m}^{2}=x f F^{\prime} / 2=x \varphi F / 2$.

Сравнивая профили касательных напряжений - рассчитанного по формуле (2.12) и определенного опытным путем в $\left[{ }^{9}\right]$, видим их удовлетворительное совпадение (рис. 5).

Формулы для среднеквадратичных значений пульсации скорости таковы:

$$
\begin{gathered}
\overline{u^{2}} / U_{m}^{2}=\chi^{2} f^{2}+\left(k F^{\prime} / \chi^{1 / 2}\right)^{2} / 3, \\
\overline{v^{2}} / U_{m}^{2}=\overline{w^{2}} / U_{m}^{2}=\chi^{2} f^{2} .
\end{gathered}
$$

В отношении результатов сравнения расчета по (2.13) с опытными данными (рис. 6) действительно все сказанное выше для плоской струи.

В заключение отметим, что расчеты на основе модели $\left[{ }^{5}\right]$, обеспечивая удовлетворительное совпадение с экспериментом, приводят к цели более простым путем, нежели расчеты на основе обычного уравнения баланса турбулентной энергии [ $\left.{ }^{4}\right]$.

\section{ЛИТ Е Р АТ У Р А}

I. А бр а м о в и ч Г. Н., Теория турбулентных струй, М., Физматгиз, 1960.

2. Хи н ц е И. О., Турбулентность, М., Физматгиз, 1963.

3. Ш ли х т и нг Г., Теория пограничного слоя, М., «Наука», 1974.

4. Дж аугаштин К. Е., Изв. АН СССР, Мех. жидкости и газа, № 3, 80-89, (1970).

5. Э п ш те й н А., Изв. АН ЭССР, Физ. Матем., 27, № 4, 413-422 (1978).

6. Reichardt, H., Gesetzmässigkeiten der freien Turbulenz, VDI-Forschungsh., № 414, Düsseldorf, 1951.

7. B r a d bury, L. J. S., J. Fluid Mech., 23, № 1, 31-64 (1965).

8. Х е с к е с тад Г., Прикл. механика, Е32, № 4, 1-17 (1965).

9. Т а унсенд А. А., Структура турбулентного потока с поперечным сдвигом, М., Изд-во иностр., лит., 1959 .

10. Wy gna nski, I., Fied ler, H., J. Fluid Mech., 38, № 3, 577-612 (1969).

Ннститут термофизики и электрофизики Академии наук Эстонской ССР
Поступила в редакцию 24/V 1978 


\section{A. EPSTEIN}

\section{KANALITE JA TORUDE TURBULENTSETE VOOLUDE ARVUTUS}

On esitatud autori varem avaldatud poolempiirilisel turbulentsusmudelil põhinev analüütiline lahend ühtlase turbulentse voolu kohta tasapinnalistes kanalites ja ümartorudes ning väljakujunenud turbulentse voolu ühtsed valemid, mis kirjeldavad keskmistatud kiiruse jaotust ja takistusteguri sõltuvust Reynoldsi arvust. Kiiruse defitsiidi üldvalem annab piirkondlikul üleminekul logaritmilise seaduspärasuse seina lähedal ja paraboolse sõltuvuse telje lähedal. Avaldatud arvutusvalemid on kooskõlas katseandmetega.

\section{A. EPSTEIN}

\section{SOME PROBLEMS OF THE FREE TURBULENCE}

The paper deals with calculation of the mean and fluctuating characteristics of free boundary layers of incompressible fluid (two-dimensional jet and wake) by means of a new semi-empirical model of turbulence, proposed by the author in $\left.{ }^{5}\right]$.

A system of equations of continuity, momentum and turbulent energy of the velocity fluctuation, primary component of written for a plane jet $((1.1)-(1.3))$ or a plane wake $((2.1)-(2.3))$ in the stage of self-preservation, is transformed into another set of ordinary differential equations - (1.9), (1.10) or $(2.8),(2.9)$, respectively, - and solved numerically at appropriate boundary conditions.

Comparison of the calculated values with experimental data of Bradbury $\left[{ }^{7}\right]$ and Heskestad $\left[{ }^{8}\right]$ for the jet flows and that of Townsend $\left[{ }^{9}\right]$ for the wake flows shows excellent agreement for the mean velocity profiles (Fig. 1, 4) and a rather good agreement for the shear stress profiles (Fig. 2,5). However, calculated profiles of the mean square values of velocity fluctuations do not agree well with the experimental data. The reason for such a disagreement lies partly in limited reliability of the experimental data itself which show quantitatively different behaviour of the same velocity fluctuation components across the boundary layer.

On the whole, calculations on the basis of the proposed model are considerably simpler than those where the conventional turbulent energy equation is used. 